\title{
Self-organized criticality: Does it have anything to do with criticality and is it useful?
}

\author{
D. L. Turcotte \\ Department of Earth and Atmospheric Sciences, Snee Hall, Cornell University, Ithaca, NY 14853, USA
}

Received: 2 June 2000 - Revised: 10 October 2000 - Accepted: 21 October 2000

\begin{abstract}
Three aspects of complexity are fractals, chaos, and self-organized criticality. There are many examples of the applicability of fractals in solid-earth geophysics, such as earthquakes and landforms. Chaos is widely accepted as being applicable to a variety of geophysical phenomena, for instance, tectonics and mantle convection. Several simple cellular-automata models have been said to exhibit selforganized criticality. Examples include the sandpile, forest fire and slider-blocks models. It is believed that these are directly applicable to landslides, actual forest fires, and earthquakes, respectively. The slider-block model has been shown to clearly exhibit deterministic chaos and fractal behaviour. The concept of self-similar cascades can explain self-organized critical behaviour. This approach also illustrates the similarities and differences with critical phenomena through association with the site-percolation and diffusionlimited aggregation models.
\end{abstract}

\section{Introduction}

The concept of self-organized criticality was proposed by Bak et al. (1988) as an explanation for the behaviour of a simple cellular-automata model that they developed. In this model, there is a square grid of boxes and at each time, step a particle is dropped into a randomly selected box. When a box accumulates four particles, the particles are redistributed to the four neighboring boxes, or in the case of edge boxes, lost from the grid. Redistributions can lead to further instabilities, with "avalanches" of particles lost from the edge of the grid. Due to of this "avalanche" behaviour, this was called a "sandpile" model. The noncumulative frequency-area distribution of model "avalanches" was found to satisfy a powerlaw (fractal) distribution:

Correspondence to: D. L. Turcotte

(turcotte@geology.cornell.edu)
$N \sim A^{-\alpha}$

where $N$ is the number of avalanches with area $A$, and $\alpha$ is a constant with a value $\alpha \approx 1$.

A second model that can exhibit self-organized critical behaviour is the slider-block model (Carlson and Langer, 1989). In this model, an array of slider-blocks are connected to a constant velocity driver plate by puller springs, and to each other, by connector springs. The blocks exhibit stick-slip behaviour due to frictional interactions with the plate when the blocks are pulled across it. The frequency-area distribution of the smaller slip events again satisfies Eq. (1) with $\alpha \approx 1$. The area $\mathrm{A}$ is defined to be the number of blocks that participate in a slip event. This model is completely deterministic, whereas the sandpile model is stochastic. This model also provides a direct bridge to deterministic chaos; for instance, Huang and Turcotte (1990) showed that a pair of slider blocks pulled over a surface can exhibit deterministic chaos with a period doubling route to chaos.

A third model that exhibits deterministic chaos is the forestfire model (Bak et al., 1992; Drossel and Schwabl, 1992). In the simplest version of this model, a square grid of sites is considered. At each time step either a tree is planted on a randomly chosen site (if the site is unoccupied) or a spark is dropped on the site. If the spark is dropped on a tree, that tree and all adjacent trees are "burned" in a model "forest fire". The frequency-area distribution of the smaller fires again satisfies Eq. (1) with $\alpha=1.0-1.2$. The area $A$ is defined to be the number of trees that are burned in a fire. The forest-fire model is closely related to the site-percolation model that is known to exhibit critical behaviour (Stauffer and Aharony, 1992). If trees are planted on a grid without fires, site-percolation behaviour is found. The critical point is reached when a tree cluster spans the grid. The frequencyarea distribution is power-law only at this critical point.

A satisfactory definition for self-organized criticality remains elusive. The three models discussed above exhibit similar behaviours, but there are also significant differences. 
Many variations on these models have been proposed; some are considered self-organized critical models, but many others are not. Totally different models have been proposed that exhibit self-organized critical behaviour. Some of these models are applicable to problems in the biological and social sciences. Bak (1996) has provided a comprehensive, yet personal review of developments. Extensive discussions of self-organized criticality have also been given by Jensen (1998) and by Turcotte (1997, 1999a). Although the concept of self-organized criticality was conceived as an explanation for simple "toy" models, it has been associated with several natural hazards (Malamud and Turcotte, 1999).

\section{Applications of the "sandpile" model}

Shortly after the "sandpile" model was proposed, a number of laboratory studies were undertaken to determine whether actual sandpiles exhibit the behaviour attributed to self-organized criticality. A variety of frequency-size statistics for avalanches were found (Nagel, 1992; Feder, 1995); in some cases, the results were consistent with the power-law relation (1) but in other cases, they were not. Actual landslides are often well represented by the power-law relation (1) (Pelletier et al., 1997; Hovius et al., 2000). Turbidite deposits are often associated with slumps (avalanches) off continental margins. Rothman et al. (1994) associate the observed power-law frequency-thickness distributions of turbidite layers with the "sandpile" model. It appears reasonable to conclude that naturally occurring landslides are an example of self-organized criticality.

\section{Applications of the slider-block model}

Since the concept of self-organized criticality was first introduced, earthquakes have been identified as an example of this phenomena in nature (Bak and Tang, 1989). For over 50 years, it has been accepted that earthquakes universally obey Gutenberg-Richter scaling; the cumulative number of earthquakes per year in a region with magnitudes greater than $m$, $\dot{N}_{C E}$, is related to $m$ by

$\log \dot{N}_{C E}=-b m+\log \dot{a}$

where the constant $b$ is known as the $b$-value and has a nearuniversal value $b=0.90 \pm 0.15$. The constant $\dot{a}$ is a measure of the intensity of the regional seismicity.

When Eq. (2) is expressed in terms of the earthquake rupture area, $A_{E}$, instead of earthquake magnitude, this relation becomes a power-law

$\log \dot{N}_{C E} \sim A_{E}^{-b}$

which is very similar to Eq. (1), except that Eq. (3) is based on cumulative statistics and Eq. (1) is based on noncumulative statistics.

With aftershocks removed, the background seismicity in southern California is nearly uniform from year to year (Tur- cotte, 1999b). Small earthquakes behave like a thermal background noise. This is observational evidence that the Earth's crust is continuously on the brink of failure (Scholz, 1991). Further evidence for this comes from induced seismicity. Whenever the crust is loaded, whether in a tectonically active area or not, earthquakes are induced. Examples of loading include the filling of a reservoir behind a newly completed dam or the high-pressure injection of fluids in a deep well.

While there are important similarities between slider-block models and earthquakes, there are also important differences. Slider-block models would be representative of a distribution of earthquakes on a single fault. However, the GutenbergRichter distribution of earthquakes is not associated with a single fault, but with a hierarchy of faults. The earthquakes in southern California occur over a broad zone with a width of about $200 \mathrm{~km}$ on a wide variety of faults associated with the San Andreas system.

There is extensive evidence that the distribution of faults in the crust is fractal (power-law). This has led to the suggestion (Sammis et al., 1987) that comminution shattering of the crust has led to a power-law distribution of tectonic blocks. The boundaries of these blocks are the power-law distribution of faults.

An important aspect of self-organized criticality relative to earthquakes is the implications for earthquake forecasting and prediction. Acceptance of the validity of the GutenbergRichter relation and the constant rate of occurrence of small earthquakes implies that the observed frequency of occurrence of small earthquakes can be extrapolated to estimate the recurrence frequencies of larger earthquakes. This is routinely done and is the primary basis for published maps of the earthquake hazard (Frankel, 1995).

\section{Applications of the forest-fire model}

An obvious application of the forest fire model is to actual forest and wild fires (Malamud et al., 1998). From this study, four forest fire and wildfire data sets from the United States and Australia were found to have frequency-area statistics that were in good agreement with the power-law relation (1). Similar results have been reported for California (Minnich and Chou, 1997). Considering the many complexities of the initiation and propagation of forest fires and wildfires, it is remarkable that the frequency-area statistics are very similar under a wide variety of environments. The proximity of combustible material varies greatly. The behaviour of a particular fire depends strongly on meteorological conditions. Firefighting efforts extinguish many fires. Despite these complexities, the application of the frequency-area distributions associated with the forest fire model appears to be robust. Forest and wildfires also appear to be an example of selforganized critical behaviour in nature. 


\section{Conclusions}

A number of models and natural phenomena have been discussed that may or may not exhibit self-organized critical behaviour. It is desirable to provide a "clean" definition of what self-organized criticality is, but there is not a universally accepted definition. In fact, some authors would submit that self-organized critical behaviour is the same as critical behaviour.

Most authors would agree that the original sandpile model proposed by Bak et al. (1988) is an example of self-organized critical behaviour. But this agreement does not extend to the slider-block and forest fire models. It is certainly possible to provide a rigorous mathematically-based definition of selforganized criticality. For example, full self-similarity would be required. However, such a restricted definition may not address the real utility of the basic concept. The essential question is whether a broad range of real complex phenomena exhibits similar behaviour under very broad conditions. This seems to be true for earthquakes, landslides, and forest fires. It may also be true for a variety of other examples in the physical, biological and social sciences. A few examples are species extinctions, epidemics, stock market crashes and wars.

A universal feature of these phenomena is that they are driven systems that involve "avalanches" with a fractal (power-law) frequency-size distribution. There is a steadystate "input" and the "output" occurs in the "avalanches." Although it has not been widely established, there is evidence that a system with self-organized criticality is on the "edge" of chaos. Adjacent solutions exhibit a power-law divergence in time, whereas chaotic solutions exhibit an exponential divergence (a positive Lyapunov exponent).

The simple forest fire model exhibits many of the characteristics associated with self-organized criticality. This forest fire model is also closely related to the site-percolation model that exhibits critical behaviour. The transient forest fire model without fires is identical to the site-percolation model; the critical point is when a cluster has formed that crosses the grid. For both the quasi-steady-state forest fire model with fires, and the transient forest fire model without fires, there is an inverse cascade of trees from small to large clusters. Clusters of trees grow by the addition of new trees and the growing clusters coalesce to form larger tree clusters. Significant numbers of trees are lost only in the fires that burn the largest clusters. In the quasi-steady-state forest fire model, this cascade gives a power-law frequency-area distribution for both the smaller clusters of trees and the forest fires (Turcotte et al., 1999). The clustering process is statistically identical to the growth of river networks and to the diffusion limited aggregation (DLA) clusters (Gabrielov et al., 1999). In the transient cascade associated with the site-percolation problem, a power-law distribution is found only at a critical density of trees. The quasi-steady-state, self-similar cascade can be extended to explain the behaviour of both the "sandpile" and slider-block models.

The above discussion has focused on the power-law (frac- tal) distributions of the "avalanches". However, there are other important aspects of the behaviour of models and natural phenomena that are associated with self-organized criticality. One of these concerns correlation lengths. Studies of critical phenomena emphasize the systematic increase in the correlation length as the critical point is approached. It has not been established whether there are systematic temporal variations in correlation length in models with selforganized criticality. There is observational evidence for a well-defined correlation length for seismic activation prior to a major earthquake (Bowman et al., 1998).

Although the concept of self-organized criticality is poorly defined, there is accumulating evidence that it is directly applicable to a variety of problems in geophysics. It is also a subject that is in an early stage of development and one that may relate phenomena over a very broad range of subjects.

Acknowledgement. The author would like to acknowledge many stimulating discussions with Jie Huang, Bill Klein, Bruce Malamud, Gleb Morein, Bill Newman, David Roberts, John Rundle, Charlie Sammis, and Lennie Smith. This research has been partially supported by NSF grant no. EAR9804859.

\section{References}

Bak, P., How Nature Works: The Science of Self-Organized Criticality, Copernicus, New York, 1996.

Bak, P., Chen, K., and Tang, C., A forest-fire model and some thoughts on turbulence, Phys. Let., A14, 297-300, 1992.

Bak, P. and Tang, C., Earthquakes as a self-organized critical phenomenon, J. Geophys. Res., 9, 15635-15637, 1989.

Bak, P., Tang, C., and Wiesenfeld, K., Self-organized criticality, Phys. Rev., A3, 364-374, 1988.

Bowman, D. D., Ouillon, G., Sammis, C. G., Sornette, A., and Sornette, D., An observational test of the critical earthquake concept, J. Geophys. Res., 10, 24359-24372, 1998.

Carlson, J. M. and Langer, J. S., Mechanical model of an earthquake fault, Phys. Rev., A4, 6470-6484, 1989.

Drossel, B. and Schwabl, F., Self-organized critical forest-fire model, Phys. Rev. Let., 6, 1629-1632, 1992.

Feder, J., The evidence for self-organized criticality in sand-pile dynamics, Fractals, 3, 431-443, 1995.

Frankel, A. F., Mapping seismic hazard in the central and eastern United States, Seis. Res. Let., 6, No. 4, 8-21, 1995.

Gabrielov, A., Newman, W. I., and Turcotte, D. L., An exactly soluble hierarchical clustering model: Inverse cascades, selfsimilarity, and scaling, Phys. Rev., E6, 5293-5300, 1999.

Hovius, N., Stark, C. P., Hao-Tsu, C., and Jinn-Chuan, L., Supply and removal of sediment in a landslide-dominated mountain belt: Central Range, Taiwan, J. Geol., 10, 73-89, 2000.

Huang, J. and Turcotte, D. L., Are earthquakes an example of deterministic chaos?, Geophys. Res. Let., 1, 223-226, 1990.

Jensen, H. J., Self-Organized Criticality: Emergent Complex Behavior in Physical and Biological Sciences, Cambridge University Press, Cambridge, 1998.

Malamud, B. D., Morein, G., and Turcotte, D. L., Forest fires: An example of self-organized critical behaviour, Science, 28, 1840 1842, 1998.

Malamud, B. D. and Turcotte, D. L., Self-organized criticality applied to natural hazards, Natural Hazards, 2, 93-116, 1999. 
Minnich, R. A. and Chou, Y. H., Wildland fire patch dynamics in the Chaparr of southern California and northern Baja California, Int. J. Wildland Fire, 7, 221-248, 1997.

Nagel, S. R., Instabilities in a sandpile, Rev. Mod. Phys., 6, 321325, 1992.

Pelletier, J. D., Malamud, B. D., Blodgett, T. A., and Turcotte, D. L., Scale-invariance of soil moisture variability and its implications for the frequency-size distribution of landslides, Eng. Geol., 4, 255-268, 1997.

Rothman, D. H., Grotzinger, J. P., and Flemings, P., Scaling in turbidite deposition, J. Sed. Petrol., A6, 59-67, 1994.

Sammis, C., King, G., and Biegel, R. The kinematics of gouge deformation, Pure Ap. Geophys., 12, 777-812, 1987.

Scholz, C. H., Earthquakes and faulting: Self-organized critical phenomena with a characteristic dimension, in Spontaneous For- mation of Space-Time Structures and Criticality, T. Riste and D. Sherrington, eds. 41-56, Kluwer Academic Publishers, Netherlands, 1991.

Stauffer, D. and Aharony, A., Introduction to Percolation Theory, Second Edition, Taylor and Francis, London, 1992.

Turcotte, D. L., Fractals and Chaos in Geology and Geophysics, Second Edition, Cambridge University Press, Cambridge, 1997.

Turcotte, D. L., Self-organized criticality, Rep. Prog. Phys., 6, 1377-1429, 1999a.

Turcotte, D. L., Seismicity and self-organized criticality, Phys. Earth Planet. Int., 11, 275-293, 1999b.

Turcotte, D. L., Malamud, B. D., Morein, G., and Newman, W. I., An inverse-cascade model for self-organized critical behaviour, Physica, A26, 629-643, 1999. 